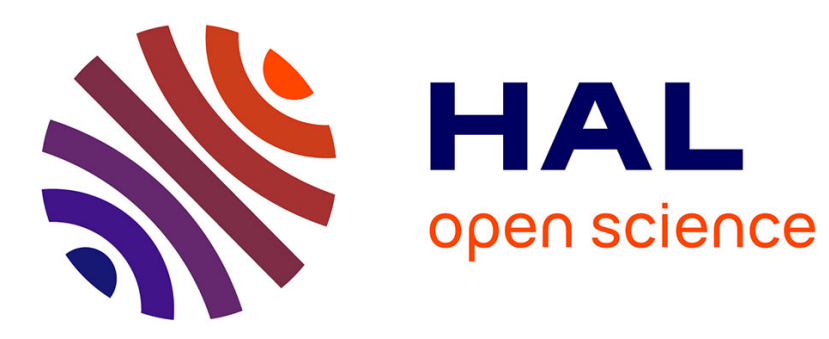

\title{
Robust Possibilistic Production Planning Under Budgeted Demand Uncertainty
}

Romain Guillaume, Adam Kasperski, Pawel Zielinski

\section{To cite this version:}

Romain Guillaume, Adam Kasperski, Pawel Zielinski. Robust Possibilistic Production Planning Under Budgeted Demand Uncertainty. IEEE International Conference on Fuzzy Systems (FUZZ-IEEE 2020), IEEE Computational Intelligence Society (CIS), Jul 2020, Glasgow (virtual), United Kingdom. 10.1109/FUZZ48607.2020.9177596 . hal-03272128

\section{HAL Id: hal-03272128 \\ https://hal.science/hal-03272128}

Submitted on 28 Jun 2021

HAL is a multi-disciplinary open access archive for the deposit and dissemination of scientific research documents, whether they are published or not. The documents may come from teaching and research institutions in France or abroad, or from public or private research centers.
L'archive ouverte pluridisciplinaire HAL, est destinée au dépôt et à la diffusion de documents scientifiques de niveau recherche, publiés ou non, émanant des établissements d'enseignement et de recherche français ou étrangers, des laboratoires publics ou privés. 


\section{Robust Possibilistic Production Planning Under Budgeted Demand Uncertainty}

\author{
Romain Guillaume \\ Université de Toulouse-IRIT \\ Toulouse, France \\ romain.guillaume@irit.fr
}

\author{
Adam Kasperski \\ Wroctaw University of Science \\ and Technology \\ Wrocław, Poland \\ adam.kasperski@pwr.edu.pl
}

\author{
Paweł Zieliński \\ Wroctaw University of Science \\ and Technology \\ Wrocław, Poland \\ pawel.zielinski@pwr.edu.pl
}

\begin{abstract}
The paper deals with a production planning problem, that is a version of the capacitated single-item lot sizing problem with backordering, under uncertain cumulative demands, modeled by fuzzy intervals centered around the cumulative demand nominal values. Their membership functions are regarded as possibility distributions for the values of the unknown cumulative demands. Furthermore, the budgeted uncertainty model is assumed, in which at most a specified number of cumulative demands can deviate from their nominal values at the same time. In order to choose a robust production plan that optimizes against plausible cumulative demand scenarios, under the model assumed, possibilistic criteria are adopted. Polynomial linear programming based methods for finding such robust production plans are proposed, showing in this way that the problem under consideration is not much computationally harder than its deterministic counterpart. Some results of computational tests are presented.
\end{abstract}

Index Terms - production planning, demand uncertainty, fuzzy interval, possibility theory

\section{INTRODUCTION}

Nowadays most companies use the manufacturing resource planning method (MRP II) to plan their production, which is composed of three levels [1]: the strategic level (Sales and Operation Plan-S\&OP), the tactical level (Master Production Scheduling (MPS) and Material Requirement Planning (MRP)) and the operational level (detailed scheduling and shop floor control). We will be concerned with the tactical level under uncertainty. Ill-known data in this context, for instance: demand, lead time capacities, etc., induce risks for the manufacturer such as backordering and obsolete inventory. Therefore, taking into account the uncertainty in the planning process is a very important issue, since it allows the decision maker to reduce these risks. Several extensions of MRP II have been proposed in the literature in order to cope with ill-known data, among others, those where uncertain demands in periods (MPS and MRP) [2]-[4], uncertain demands in periods and orders (MRP) [5] and the imprecision in order quantities and dates with uncertain order (MRP) [6] are considered (see [7] for a comprehensive review).

Romain Guillaume has benefitted from the AI Interdisciplinary Institute ANITI funding. ANITI is funded by the French "Investing for the Future PIA3" program under the Grant agreement no. ANR-19-PI3A-0004.

Adam Kasperski and Paweł Zieliński were supported by the National Science Centre, Poland, grant 2017/25/B/ST6/00486.
In this paper we wish to investigate a version of the capacitated single-item lot sizing problem (CLSP) with backordering under uncertainty in the demand, assuming that its deterministic counterpart is polynomially solvable (see, e.g. [8]). Typically, the uncertainty in parameters is modeled by specifying a set, denoted by $\mathcal{U}$, of all possible realizations of the parameters (demands), called scenarios. When there is no probability distribution in scenario set $\mathcal{U}$, the commonly used methods of defining $\mathcal{U}$ are the discrete and interval representations (see, e.g., [9]). In this case, in order choose a robust production plan, the minmax criterion is usually applied. Unfortunately, for the discrete representation of demands the CLSP problem with the minmax criterion turned out to be NP-hard even for two demand scenarios [9]. A situation is computationally much better for the interval representation of demands [10], [11].

We focus on a generalization of the interval representation which additionally collects plausible demand scenarios. Namely, we model uncertain cumulative demands by fuzzy intervals, centered around their nominal values, regarded as possibility distributions for the demand. Using possibility theory [12] one can compute a possibility distribution in the cumulative demand scenario set $\mathcal{U}$ induced by these fuzzy intervals. It is worth pointing out that we consider uncertainty in the cumulative demands instead of the per-period demands, because such uncertainty representation is more realistic than the one in the demands in periods, which can lead to huge uncertainty over the planning horizon. Following [13], [14], we additionally assume the budgeted uncertainty model, in which at most a specified number of the cumulative demands, denoted by $\Gamma$, can deviate from their nominal values at the same time. In this case, a reliable production plan seems to be a robust one that optimizes against plausible scenarios in $\mathcal{U}$, in which at most $\Gamma$ cumulative demands take values different from their nominal ones. Since the worst case cost of such robust plan can be significantly higher than the cost of an optimal plan under the nominal scenario, we wish to choose among the above robust production plans, similarly as in [15], [16], the one whose costs in plausible scenarios are not too far from the nominal cost. To find such plans for our CLSP problem under uncertain cumulative demands we use in this paper necessity measure based criteria [17]. We call these plans best necessarily (soft) feasible production plans. For a 
deeper discussion of possibility theory in optimization we refer the reader to [18].

We provide polynomial methods, that are an extension to the fuzzy case an interval one given in [19], for computing best necessarily (soft) feasible production plans. We show in this way that introducing uncertainty in the cumulative demands in the possibilistic setting does not make our problem much computationally harder than its deterministic counterpart. Furthermore, the computational experiments performed suggest that taking additional information about possibility distributions of the cumulative demands given by fuzzy intervals into account may lead to production plans with a better quality over a set of plausible scenarios.

\section{Production Planning Problem}

In this section we formulate a production planning problem that we examine in the paper. It is a version of the well-known capacitated single-item lot sizing problem with backordering (see, e.g., [8]). We first state the problem with precise parameters. Then we assume that demands are subject to uncertainty - the rest of the parameters are precisely known.

\section{A. Deterministic Problem}

We are given $T$ periods, a demand $d_{t}$ in each period $t$, $t \in[T]$ ([T] denotes the set $\{1, \ldots, T\}$ ), production, inventory and backordering costs and a selling price, denoted by $c^{P}, c^{I}$, $c^{B}$ and $b^{P}$, respectively, which do not depend on period $t$. We assume zero initial inventory and initial backorder. The problem is to find a feasible production amount $x_{t}$ in each period $t$, called production plan, subject to the condition of satisfying each demand, which minimizes the total storage and backordering costs minus the benefit from selling the product.

Let us denote by $\mathbb{X}$ the set of production plans. We assume that $\mathbb{X}$ is specified by some linear constraints, for example

$$
\begin{aligned}
\mathbb{X}=\left\{\boldsymbol{x}=\left(x_{t}\right)_{t \in[T]} \in \mathbb{R}_{+}^{T}: x_{t}\right. & \geq 0, l_{t} \leq x_{t} \leq u_{t}, \\
L_{t} & \left.\leq \sum_{i \in[t]} x_{i} \leq U_{t}, t \in[T]\right\},
\end{aligned}
$$

where $l_{t}, u_{t}$ and $L_{t}, U_{t}$ are given capacity and cumulative capacity limits, respectively.

Our problem can be modeled by the following linear program:

$$
\begin{aligned}
& \min \sum_{t \in[T]}\left(c^{I} I_{t}+c^{B} B_{t}+c^{P} x_{t}-b^{P} s_{t}\right) \\
& \text { s.t. } B_{t}-I_{t}=D_{t}-X_{t} \\
& \sum_{i \in[t]} s_{i}=D_{t}-B_{t} \\
& t \in[T], \\
& B_{t}, I_{t}, s_{t} \geq 0 \\
& t \in[T], \\
& x \in \mathbb{X} \subseteq \mathbb{R}_{+}^{T} \text {, } \\
& t \in[T],
\end{aligned}
$$

where $D_{t}=\sum_{i \in[t]} d_{i}$ and $X_{t}=\sum_{i \in[t]} x_{i}, D_{t}$ and $X_{t}$ stand for the cumulative demand up to period $t$ and the cumulative production up to period $t$, respectively.
An easy computation shows that (1)-(5) can be rewritten in the following equivalent compact form, which is more convenient to analyze:

$$
\begin{array}{r}
\min _{\boldsymbol{x} \in \mathbb{X}} \mathcal{C}(\boldsymbol{x}, \boldsymbol{D})=\min _{\boldsymbol{x} \in \mathbb{X}} \sum_{t \in[T]} \max \left\{c^{I}\left(X_{t}-D_{t}\right), c^{B}\left(D_{t}-X_{t}\right)\right\} \\
+c^{P} X_{T}-b^{P} \min \left\{X_{T}, D_{T}\right\},
\end{array}
$$

where $\boldsymbol{D}=\left(D_{t}\right)_{t \in[T]}$ is a $T$-vector of cumulative demands.

\section{B. Problem Under Uncertainty}

We now admit that demands are subject to uncertainty. In practice, knowledge about uncertainty in a demand is expressed as $\pm \Delta$, where $\Delta$ is a possible deviation from its nominal value, which means that the actual demand will take some value within the interval (the support) given, but it is not possible at present to predict which one. In consequence, it induces a natural interval uncertainty representation (see, e.g., [9]). A demand has a twofold interpretation, namely, a demand in a period, $d_{t}$, or a cumulative demand, $D_{t}, t \in[T]$. The former interpretation is often assumed. However, in this case and under the interval uncertainty $\Delta$ cumulates in the subsequent periods due to the interval addition, $\widetilde{D}_{t}=\widetilde{\sum}_{t \in[T]} \tilde{d}_{t}$, which is not realistic. Accordingly, we assume that the uncertainty of demand $\tilde{d}_{t}$ will lie in the cumulative demand $\widetilde{D}_{t}$ modeled by an interval or a fuzzy interval prescribed, i.e. an interval with information about a set of plausible cumulative demand values (see, e.g., [12]).

From now on we will be concerned with a version of (6) with uncertain cumulative demands $\widetilde{\boldsymbol{D}}=\left(\widetilde{D}_{t}\right)_{t \in[T]}$ :

$$
\begin{array}{r}
\widetilde{\min _{\boldsymbol{x}}} \mathcal{C}(\boldsymbol{x}, \widetilde{\boldsymbol{D}})=\widetilde{\min _{\boldsymbol{x} \in \mathbb{X}}} \sum_{t \in[T]} \max \left\{c^{I}\left(X_{t}-\widetilde{D}_{t}\right), c^{B}\left(\widetilde{D}_{t}-X_{t}\right)\right\} \\
+c^{P} X_{T}-b^{P} \min \left\{X_{T}, \widetilde{D}_{T}\right\} .
\end{array}
$$

The precise models of the cumulative demand uncertainty, $\widetilde{D}$, introduced here, will be given in Sec. III and IV-A.

\section{Robust PRoduction Plans Under InTERVAL UNCERTAINTY}

In this section we proceed with the study of the problem (7) and modeling uncertain cumulative demands in the setting of the interval budgeted uncertainty representation proposed in [13], [14]. Namely, we assume that $\widetilde{D}_{t}, t \in[T]$, is a random variable, symmetrically distributed around its nominal (expected) value $\widehat{D}_{t}$. Thus the value of $\widetilde{D}_{t}$ is only known to belong to the support $\left[\widehat{D}_{t}-\Delta_{t}, \widehat{D}_{t}+\Delta_{t}\right]$ of $\widetilde{D}_{t}$, where $\Delta_{t}$ is its the maximum deviation from its nominal value. Furthermore an integer parameter in $[0, T]$, denoted by $\Gamma$ and called a robustness level or a budget, that specifies the maximal number of cumulative demands, whose values can be different from their nominal ones is prescribed. Let

$$
\mathcal{D}=\left\{\boldsymbol{D}=\left(D_{t}\right)_{t \in[T]} \in \mathbb{R}^{T}:\left|\left\{t: D_{t} \neq \widehat{D}_{t}\right\}\right| \leq \Gamma\right\} .
$$

We define $\mathcal{U}$ to be the Cartesian product of the supports, i.e.

$$
\mathcal{U}=\left[\widehat{D}_{1}-\Delta_{1}, \widehat{D}_{1}+\Delta_{1}\right] \times \cdots \times\left[\widehat{D}_{T}-\Delta_{T}, \widehat{D}_{T}+\Delta_{T}\right] .
$$


Throughout the paper, we assume that the supports are nonoverlapping, i.e. $\widehat{D}_{t}+\Delta_{t} \leq \widehat{D}_{t+1}-\Delta_{t+1}, t \in[T-1]$. This assumption is realistic, in particular at the tactical level of planning, for instace Master Production Schedule (MPS) problems (see, e.g. [1]), where the lengths of periods are big enough (for example, they are equal to one month). Hence for each cumulative demand scenario $D$, the inequalities $D_{t} \leq D_{t+1}, t \in[T-1]$, hold. Thus the set of feasible cumulative demand scenarios, according to the model in [13], [14], is the intersection of the scenario sets (8) and (9), respectively $(\mathcal{D} \cap \mathcal{U})$. In order to compute a robust production plan, similarly to [13], [14], a minmax approach is adopted (see, e.g., [9]). This leads to the following problem:

$$
\min _{\boldsymbol{x} \in \mathbb{X}} \max _{\boldsymbol{D} \in \mathcal{D} \cap \mathcal{U}} \mathcal{C}(\boldsymbol{x}, \boldsymbol{D})
$$

An optimal production plan $x^{*}$ to (10), called robust, is the one whose the maximum cost $\mathcal{C}\left(\boldsymbol{x}^{*}, \boldsymbol{D}\right)$ is minimum over scenario set $\mathcal{D} \cap \mathcal{U}$. Indeed, such solution computed is a robust choice, because we are sure that it optimizes against all scenarios, in which at most $\Gamma$ cumulative demands take values different from their nominal ones. Moreover, by changing the value of $\Gamma$, from 0 to $T$, one can flexibly control the level of robustness of a resulting plan, namely, if $\Gamma=0$, then all the cumulative demands take their nominal values $\widehat{D}_{t}, t \in[T]$, and we arrive to the deterministic problem (6), so we have one scenario and the uncertainty is ignored. On the other hand, when $\Gamma=T$, all the cumulative demands are uncertain and thus a production plan found is strictly robust and can be highly conservative, because we need to consider all possible cumulative demand scenarios in $\mathcal{U}$.

Fortunately, finding a robust production plan $\boldsymbol{x}^{*}$, i.e. solving the problem (10), can be reduced to the following linear programming problem (see [19] for details), so it is polynomially solvable:

$$
\begin{aligned}
& \min \sum_{t \in[T]} \pi_{t}+c^{P} X_{T}+\Gamma \alpha+\sum_{t \in[T]} \gamma_{t} \\
& \text { s.t. } \pi_{t} \geq c^{I}\left(X_{t}-\widehat{D}_{t}\right) \text {, } \\
& t \in[T-1], \\
& \pi_{t} \geq c^{B}\left(\widehat{D}_{t}-X_{t}\right), \\
& t \in[T-1] \text {, } \\
& \pi_{T} \geq c^{I}\left(X_{T}-\widehat{D}_{T}\right)-b^{P} \widehat{D}_{T}, \\
& \pi_{T} \geq c^{B}\left(\widehat{D}_{T}-X_{T}\right)-b^{P} X_{T}, \\
& \alpha+\gamma_{t} \geq c^{I}\left(X_{t}-\left(\widehat{D}_{t}-\Delta_{t}\right)\right)-\pi_{t}, \quad t \in[T-1], \\
& \alpha+\gamma_{t} \geq c^{B}\left(\widehat{D}_{t}+\Delta_{t}-X_{t}\right)-\pi_{t}, \quad t \in[T-1], \\
& \alpha+\gamma_{T} \geq c^{I}\left(X_{T}-\left(\widehat{D}_{T}-\Delta_{T}\right)\right) \\
& -b^{P}\left(\widehat{D}_{T}-\Delta_{T}\right)-\pi_{T}, \\
& \alpha+\gamma_{T} \geq c^{B}\left(\widehat{D}_{T}+\Delta_{T}-X_{T}\right) \\
& -b^{P} X_{T}-\pi_{T} \text {, } \\
& \alpha, \gamma_{t} \geq 0, \pi_{t} \text { unrestricted } \\
& x \in \mathbb{X} \text {, }
\end{aligned}
$$

where the value of the objective function (11) equals $\max _{\boldsymbol{D} \in \mathcal{D} \cap \mathcal{U}} \mathcal{C}\left(\boldsymbol{x}^{*}, \boldsymbol{D}\right)$. It is worth pointing out that the ap- proach here considered, based on the interval budgeted uncertainty representation [13], [14] and the minmax criterion, exploits only information contained in the supports of the uncertain cumulative demands. It does not take any additional information about the cumulative demand distributions into account. In the next section we apply a more elaborate approach (see, e.g., [17]) for dealing with cumulative demand uncertainty that consists in collecting both supports and plausible demand values and extends the results presented in this section.

\section{Robust Production Plans Under Possibilistic UNCERTAINTY}

In this section we apply more elaborate approach to model uncertain cumulative demands $\widetilde{D}_{t}, t \in[T]$, in which they are modeled by fuzzy intervals whose membership functions are regarded as possibility distributions, representing the sets of plausible values of uncertain cumulative demands. In consequence, we give a possibilistic counterpart of problem (7) and, as a byproduct, we extend the model based on the interval budgeted uncertainty representation presented in Sec. III to the fuzzy case. In order to find robust production plans under the possibilistic uncertainty, that takes into account an information about cumulative demand distributions while a plan is computed, we adopt the concept of best necessarily (soft) feasible solutions proposed in [17] for linear programming problems.

\section{A. Possibilistic Model of Uncertainty}

Consider uncertain cumulative demands $\widetilde{D}_{t}, t \in[T]$. In the interval model uncertainty (see Sec. III), we only know the support $\left[\widehat{D}_{t}-\Delta_{t}, \widehat{D}_{t}+\Delta_{t}\right]$. This means that $\widetilde{D}_{t}$ will take some value within the support but it is not possible at present to predict which one. However, in practice the information about plausibility of values of $\widetilde{D}_{t}$ can be exploited in computing production plans with a better quality. In the model assumed in this section $\widetilde{D}_{t}$ is a fuzzy interval, whose membership function is continuous, symmetrically distributed around the nominal value $\widehat{D}_{t}$ and the support equal to $\left[\widehat{D}_{t}-\Delta_{t}, \widehat{D}_{t}+\Delta_{t}\right]$ (see for instance Fig. 1a). The membership function $\mu_{\widetilde{D}_{t}}$ of fuzzy interval $\widetilde{D}_{t}$ is regarded as a possibility distribution for $\widetilde{D}_{t}$, denoted by $\pi_{\widetilde{D}_{t}}$, which describes the set of more or less plausible values of $\widetilde{D}_{t}$. The value of $\mu_{\widetilde{D}_{t}}\left(D_{t}\right)$ represents the possibility degree of the assignment $\widetilde{D}_{t}=D_{t}$, i.e.

$$
\Pi\left(\widetilde{D}_{t}=D_{t}\right)=\pi_{\widetilde{D}_{t}}\left(D_{t}\right)=\mu_{\widetilde{D}_{t}}\left(D_{t}\right) .
$$

where $\Pi\left(\widetilde{D}_{t}=D_{t}\right)$ is the possibility of the event that $\widetilde{D}_{t}$ will take the value of $D_{t}$. A possibility degree can be viewed as an upper bound on a probability degree (see [12], [20] for more details).

We recall that the set $\mu_{\widetilde{D}_{t}}^{\lambda}=\left\{D_{t} \in \mathbb{R}: \mu_{\widetilde{D}_{t}}\left(D_{t}\right) \geq \lambda\right\}$, $\lambda \in(0,1]$, is called a $\lambda$-cut of $\widetilde{D}_{t}$, and $\widetilde{D}_{t}^{0}$ is the support of $\widetilde{D}_{t}$. Similarly as in Sec. III, we assume that the supports are nonoverlapping. The $\lambda$-cuts of $\widetilde{D}_{t}, \lambda \in[0,1]$, are nested closed intervals centered around the nominal value $\widehat{D}_{t}$. Thus $\widetilde{D}_{t}$ can be equivalently represented as a family of $\lambda$-cuts, i.e. 


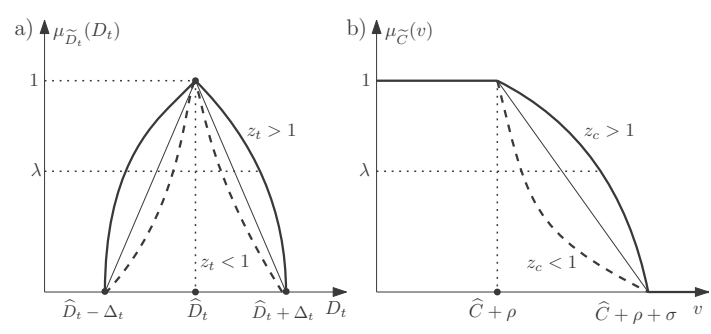

Fig. 1. a) Symmetric fuzzy interval, where $\widetilde{D}_{t}^{\lambda}=\left[\widehat{D}_{t}-\alpha_{t}(\lambda), \widehat{D}_{t}+\alpha_{t}(\lambda)\right]$, $\alpha_{t}(\lambda)=\Delta_{t} \cdot\left(1-\lambda^{z_{t}}\right), z_{t} \geq 0$. b) Fuzzy set $\widetilde{C}$ with $\mu_{\widetilde{C}}^{-1}(\lambda)=\widehat{C}+\rho+\xi(\lambda)$, $\xi(\lambda)=\sigma \cdot\left(1-\lambda^{z_{c}}\right), z_{c} \geq 0$, representing the right hand side of the flexible cost constraint.

$\widetilde{D}_{t}^{\lambda}=\left[\widehat{D}_{t}-\alpha_{t}(\lambda), \widehat{D}_{t}+\alpha_{t}(\lambda)\right], \lambda \in[0,1]$, where $\alpha_{t}(\lambda)$ is a continuous, nonincreasing function in $[0,1]$, such that $\alpha_{t}(0)=\Delta_{t}$. These cuts can be seen as the nested confidence intervals of all possible values of $\widetilde{D}_{t}$ (see [12] for methods for constructing possibility distributions of uncertain quantities). Notice also that the above representation contains a symmetric triangular fuzzy interval if $\alpha_{t}(\lambda)=\Delta_{t} \cdot(1-\lambda)$ as a special case. We can also use more general representation, namely $\alpha_{t}(\lambda)=\Delta_{t} \cdot\left(1-\lambda^{z_{t}}\right), z_{t}>0$. Indeed, the smaller the value of $z_{t}$, the less uncertainty is associated with $\widetilde{D}_{t}$ and for large $z_{t}, \widetilde{D}_{t}$ tends to a closed interval.

Let $\boldsymbol{D}=\left(D_{t}\right)_{t \in[T]} \in \mathbb{R}^{T}$ be a scenario describing a realization (a state of the world) of uncertain cumulative demands $\widetilde{\boldsymbol{D}}=\left(\widetilde{D}_{t}\right)_{t \in[T]}$ in the objective function of (7). Thus the event " $\widetilde{D}=D$ " holds. The degree of possibility that scenario $D$ will occur is provided by the following joint possibility distribution $\pi_{\widetilde{D}}$ on the set of all possible scenarios, induced by possibility distributions $\pi_{\widetilde{D}_{t}}$, (see, e.g., [21]):

$$
\begin{aligned}
\pi_{\widetilde{\boldsymbol{D}}}(\boldsymbol{D}) & =\Pi\left(\wedge_{t \in[T]}\left(\widetilde{D}_{t}=D_{t}\right)\right)=\min _{t \in[T]} \Pi\left(\widetilde{D}_{t}=D_{t}\right) \\
& =\min _{t \in[T]} \mu_{\widetilde{D}_{t}}\left(D_{t}\right) .
\end{aligned}
$$

Obviously, $\Pi(\widetilde{\boldsymbol{D}}=\boldsymbol{D})=\pi_{\widetilde{\boldsymbol{D}}}(\boldsymbol{D})$. We can now determine the set of all scenarios whose possibility of occurrence is at least $\lambda \in(0,1]$ as follows:

$$
\mathcal{U}^{\lambda}=\left\{\boldsymbol{D} \in \mathbb{R}^{T}: \pi_{\widetilde{\boldsymbol{D}}}(\boldsymbol{D}) \geq \lambda\right\}=\widetilde{D}_{q}^{\lambda} \times \widetilde{D}_{2}^{\lambda} \times \cdots \times \widetilde{D}_{T}^{\lambda}
$$

and $\mathcal{U}^{0}=\widetilde{D}_{1}^{0} \times \cdots \times \widetilde{D}_{T}^{0}$.

\section{B. Best necessarily (soft) feasible Production Plans}

We now apply the possibilistic model given in Sec. IV-A to handle the uncertainty of cumulative demands $\widetilde{D}=\left(\widetilde{D}_{t}\right)_{t \in[T]}$ in the objective function of (7). Likewise (see [13], [14] and Sec. III), we introduce a robustness level $\Gamma$, an integer in $[0, T]$. Following approach proposed [17], we will take into account, while constructing a robust production plan, the information about a possibility distribution of cumulative demand scenarios (see (22)). This allows us to find a robust plan, i.e. the one that optimizes against plausible scenarios, in which at most $\Gamma$ cumulative demands take values different from their nominal ones. Furthermore, since the worst case cost of such robust plan found can be significantly higher than the cost of an optimal plan under the nominal scenario $\widehat{D}$, we wish to choose among these robust production plans the one whose costs in plausible scenarios are not too far from the cost of an optimal plan in the nominal scenario denoted by $\widehat{C}$. Thus our production plan is a compromise one. This is an extension of the idea that comes from [15], [16]. Therefore, we are given a tolerance $\rho \in \mathbb{R}_{+}$, which reflects an acceptable increase in the cost of a plan above the nominal cost $\widehat{C}$. We say that a given production plan $\boldsymbol{x} \in \mathbb{X}$ is $\Gamma$-feasible in scenario $\boldsymbol{D} \in \mathcal{D}$ if a cost constraint of the type $\mathcal{C}(\boldsymbol{x}, \boldsymbol{D}) \leq \widehat{C}+\rho$ is satisfied, where $\mathcal{D}$ is defined as (8). We can now compute the possibility of the event that plan $x$ is $\Gamma$-feasible:

$$
\Pi(\boldsymbol{x} \text { is } \Gamma \text {-FEAS })=\sup _{\{\boldsymbol{D} \in \mathcal{D}: \mathcal{C}(\boldsymbol{x}, \boldsymbol{D}) \leq \widehat{C}+\rho\}} \pi_{\widetilde{\boldsymbol{D}}}(\boldsymbol{D}) .
$$

Applying the duality between the possibility and necessity measures (see, e.g., [21]) gives the degree of necessity of the event that plan $\boldsymbol{x}$ is $\Gamma$-feasible:

$$
\begin{aligned}
\mathrm{N}(\boldsymbol{x} \text { is } \Gamma \text {-FEAS }) & =1-\Pi(\boldsymbol{x} \text { is not } \Gamma \text {-FEAS }) \\
& =1-\sup _{\{\boldsymbol{D} \in \mathcal{D}: \mathcal{C}(\boldsymbol{x}, \boldsymbol{D})>\widehat{C}+\rho\}} \pi_{\widetilde{\boldsymbol{D}}}(\boldsymbol{D}) .
\end{aligned}
$$

$\Pi(x$ is $\Gamma$-FEAS $) \geq \lambda, \lambda \in(0,1]$, means the existence of at least one scenario $\boldsymbol{D} \in \mathcal{D}$ such that $\pi_{\widetilde{D}}(\boldsymbol{D}) \geq \lambda$ and the inequality $\mathcal{C}(\boldsymbol{x}, \boldsymbol{D}) \leq \widehat{C}+\rho$ holds ( $\boldsymbol{x}$ is $\Gamma$-feasible). While $\mathrm{N}(\boldsymbol{x}$ is $\Gamma$-FEAS $) \geq 1-\lambda, \lambda \in(0,1]$, stands for that for every scenario $\boldsymbol{D} \in \mathcal{D}$ such that $\pi_{\widetilde{\boldsymbol{D}}}(\boldsymbol{D}) \geq \lambda$ the cost constraint $\mathcal{C}(\boldsymbol{x}, \boldsymbol{D}) \leq \widehat{C}+\rho$ is satisfied. Clearly this constraint is $\Gamma$ protected, i.e. it is protected against all the scenarios above described. Furthermore, if $\mathrm{N}(\boldsymbol{x}$ is $\Gamma$-FEAS $) \geq 1-\lambda$, then $x$ is $\Gamma$-feasible with a probability at least $1-\lambda$ due to the probability-possibility consistency (see, e.g., [20], [22]).

Accordingly, within the possibilistic setting, a best production plan seems to be the one with the maximal degree of necessary $\Gamma$-feasibility (25), i.e. the most robust plan among these for which the cost constraint is $\Gamma$-protected. Such plan is optimal to the following problem:

$$
\max _{\boldsymbol{x} \in \mathbb{X}} \mathrm{N}(\boldsymbol{x} \text { is } \Gamma \text {-FEAS })
$$

This solution is called a best necessarily feasible plan. The problem (26) is a generalization to the fuzzy (possibilistic) case of the problem (10) with some ideas from [15], [16]. Its equivalent form is as follows:

$$
\begin{aligned}
\max & (1-\lambda) \\
\text { s.t. } & \mathrm{N}(\boldsymbol{x} \text { is } \Gamma \text {-FEAs }) \geq 1-\lambda, \\
& \boldsymbol{x} \in \mathbb{X} .
\end{aligned}
$$

Repeating the aforementioned interpretation of the constraint (28) and using (23) allows us to express (28) in terms of $\lambda$-cuts. This leads to the following problem:

$$
\begin{array}{ll}
\max & (1-\lambda) \\
\text { s.t. } & \max _{\boldsymbol{D} \in \mathcal{D} \cap \mathcal{U}^{\lambda}} \mathcal{C}(\boldsymbol{D}, \boldsymbol{x}) \leq \widehat{C}+\rho, \\
& \boldsymbol{x} \in \mathbb{X} .
\end{array}
$$


Observe that evaluating the left hand side of (31) for fixed $\lambda$ boils down to solving the problem (10) under the interval uncertainty. Applying (11)-(21) yields a mathematical programming formulation of the problem (26):

$$
\begin{aligned}
& (1-\lambda) \rightarrow \max \\
& \sum_{t \in[T]} \pi_{t}+c^{P} X_{T} \\
& +\Gamma \alpha+\sum_{t \in[T]} \gamma_{t} \leq \widehat{C}+\rho, \\
& \text { Constraints }(12)-(15), \\
& \alpha+\gamma_{t} \geq c^{I}\left(X_{t}-\left(\widehat{D}_{t}-\alpha_{t}(\lambda)\right)\right)-\pi_{t}, \quad t \in[T-1] \\
& \alpha+\gamma_{t} \geq c^{B}\left(\widehat{D}_{t}+\alpha_{t}(\lambda)-X_{t}\right)-\pi_{t}, \quad t \in[T-1] \\
& \alpha+\gamma_{T} \geq c^{I}\left(X_{T}-\left(\widehat{D}_{T}-\alpha_{T}(\lambda)\right)\right) \\
& -b^{P}\left(\widehat{D}_{T}-\alpha_{T}(\lambda)\right)-\pi_{T}, \\
& \alpha+\gamma_{T} \geq c^{B}\left(\widehat{D}_{T}+\alpha_{T}(\lambda)-X_{T}\right) \\
& -b^{P} X_{T}-\pi_{T}, \\
& \alpha, \gamma_{t} \geq 0, \pi_{t} \text { unrestricted } \quad t \in[T], \\
& x \in \mathbb{X}, \\
& 0 \leq \lambda \leq 1,
\end{aligned}
$$

The model (33)-(42) is nonlinear due to the terms $\alpha_{t}(\lambda)$, $t \in[T]$. However, a best necessarily feasible plan can found efficiently. We use a similar approach to that in [17] for a method for finding a best necessarily feasible solution to a linear programming problem with fuzzy coefficients. Observe that for a fixed value of $\lambda \in[0,1]$, the constraints (36)-(39) are linear. Let $\mathbb{X}^{\lambda} \subseteq \mathbb{X}$ be the set of feasible solutions to (34)(41) for a fixed value of $\lambda \in[0,1]$. Since $\alpha_{t}(\lambda), t \in[T]$, are nonincreasing $\mathbb{X}^{\lambda_{1}} \subseteq \mathbb{X}^{\lambda_{2}}$ if $\lambda_{1} \leq \lambda_{2}$. Hence (33)-(42) boils down to computing, by a binary search in $[0,1]$, the smallest value $\lambda_{\min } \in[0,1]$ for which $\mathbb{X}^{\lambda_{\min }} \neq \emptyset$ and the corresponding solution $x^{*} \in \mathbb{X}^{\lambda_{\min }}$ is a best necessarily feasible plan such that $\mathrm{N}\left(\boldsymbol{x}^{*}\right.$ is $\Gamma$-FEAS $)=1-\lambda_{\min }$. This requires checking the feasibility of (34)-(41) at most $\left\lceil\log \epsilon^{-1}\right\rceil$ times, where $\epsilon>0$ is a given accuracy. It can be done in polynomial time if the values of $\alpha_{t}(\lambda), t \in[T]$, are computed in polynomial time as well.

Fortunately, we can give a compact linear programming formulation of (26) and thus (33)-(42) for instance if the uncertainty of the cumulative demands are modeled by general $L-R$ fuzzy intervals (see, e.g., [12]) whose the left and right shape functions, $L_{t}$ and $R_{t}$, respectively, are such that $L_{t}=R_{t}=L$ for every $t \in[T]$. In our case $\alpha_{t}(\lambda)=\Delta_{t} \cdot\left(1-\lambda^{z_{t}}\right)$ (see Fig. 1a). Assume that $z_{t}=z$ for every $t \in[T]$ and $z>0$ (case $z=0$ is trivial). Thus the shape function $1-\lambda^{z_{t}}$ is the same for each demand. Now, it is sufficient to replace the term $\alpha_{t}(\lambda)$ with $\Delta_{t} \cdot \Theta$ in constraints (36)-(39), where $\Theta$ is a new variable, the objective function (33) with $\Theta$ and constraint (42) with $0 \leq \Theta \leq 1$. Clearly, the modified formulation (33)-(42) is a linear program. Let $\Theta_{\max }$ and $x^{*}$ be a part of an optimal solution to the modified (33)-(42). Now, $\lambda_{\min }=\left(1-\theta_{\max }\right)^{1 / z}$ and $\mathrm{N}\left(\boldsymbol{x}^{*}\right.$ is $\Gamma$-FEAS $)=1-\lambda_{\min }$. It is worth pointing out that the $x^{*}$ computed is the same best necessarily feasible plan for every $z>0$, of course, with different degree of necessary $\Gamma$-feasibility.

We now apply an idea of a best necessary soft feasibility proposed in [17], which is a generalization of the idea of a best necessary feasibility, already used in this section. It describes, in a more general manner, requirements on a production plan under the possibilistic uncertainty representation of cumulative demands as a plan among these robust production plans whose costs in plausible cumulative demand scenarios satisfy a flexible cost constraint. We required previously that $\mathcal{C}(\boldsymbol{x}, \widetilde{\boldsymbol{D}}) \leq \widehat{C}+\rho$ for $\boldsymbol{x} \in \mathbb{X}$, where the uncertainty of $\widetilde{D}$ is modeled by a possibility distribution $\pi_{\widetilde{D}}$ provided in (22), $\widehat{C}$ is the cost of an optimal production plan under the nominal demand scenario and $\rho \in \mathbb{R}_{+}$is a given acceptable deviation in costs of $x$ above the nominal cost $\widehat{C}$. We recall that a given plan $x \in \mathbb{X}$ is $\Gamma$-feasible in scenario $D \in \mathcal{D}$ if the cost constraint $\mathcal{C}(\boldsymbol{x}, \boldsymbol{D}) \leq \widehat{C}+\rho$ is satisfied. We replace this constraint with a flexible cost constraint: "C $(\boldsymbol{x}, \boldsymbol{D}) \widetilde{\leq} \widetilde{C}$ ", where $\widetilde{C}$ is a fuzzy set in $\mathbb{R}$ with membership function $\mu_{\widetilde{C}}$ (see for instance Fig. 1b). The value of $\mu_{\widetilde{C}}(\mathcal{C}(x, D)), D \in \mathcal{D}$, is the extent to which $\mathcal{C}(\boldsymbol{x}, \boldsymbol{D})$ satisfies the flexible cost constraint. We call such plan $x$, whose $\operatorname{cost} \mathcal{C}(\boldsymbol{x}, \boldsymbol{D})$ falls within the fuzzy right hand side $\widetilde{C}, \Gamma$-soft feasible plan in $D$. The fuzzy right hand side $\widetilde{C}$ can be seen also as a fuzzy goal. Applying possibility theory to handle both uncertainty in parameters and flexible constraints (see, e.g. [21]) we can determine the degree of necessity that a plan $x \in \mathbb{X}$ is $\Gamma$-soft feasible:

$$
\begin{aligned}
& \mathrm{N}(\boldsymbol{x} \text { is } \Gamma \text { - } \widetilde{\mathrm{FEAS}})=1-\Pi(\boldsymbol{x} \text { is not } \Gamma \text { - } \widetilde{\mathrm{FEAS}}) \\
& =1-\sup _{\boldsymbol{D} \in \mathcal{D}} \min \left\{\pi_{\widetilde{\boldsymbol{D}}}(\boldsymbol{D}), 1-\mu_{\widetilde{C}}(\mathcal{C}(\boldsymbol{x}, \boldsymbol{D}))\right\} .
\end{aligned}
$$

$\mathrm{N}(\boldsymbol{x}$ is $\Gamma$-FEAS $) \geq 1-\lambda, \lambda \in(0,1]$, means that for all scenarios $D \in \overline{\mathcal{D}}$ such that $\pi_{\widetilde{D}}(D) \geq \lambda$, the inequality $\mu_{\widetilde{C}}(\mathcal{C}(\boldsymbol{x}, \boldsymbol{D})) \geq 1-\lambda$ holds.

Hence maximizing (43) over $\mathbb{X}$ leads to the problem of finding a most robust production plan, under the possibilistic uncertainty, among ones whose costs satisfy the flexible cost constraint, fall within $\widetilde{C}$, i.e.

$$
\max _{\boldsymbol{x} \in \mathbb{X}} \mathrm{N}(\boldsymbol{x} \text { is } \Gamma \text { - } \widetilde{\mathrm{FEAS}}) \text {. }
$$

An optimal solution $x^{*}$ to (44) is called best necessarily soft feasible plan.

By a similar argument as for (28), we can rewrite (44), equivalently, in terms of $\lambda$-cuts:

$$
\begin{array}{ll}
\max & (1-\lambda) \\
\text { s.t. } & \max _{\substack{\boldsymbol{D} \in \mathcal{D} \cap \mathcal{U}^{\lambda} \\
x \in \mathbb{X}}} \mathcal{C}(\boldsymbol{D}, \boldsymbol{x}) \leq \mu_{\widetilde{C}}^{-1}(1-\lambda), \\
\quad \boldsymbol{x} \in \mathbb{2} &
\end{array}
$$

where $\mu_{\widetilde{C}}^{-1}(\lambda)=\sup \left\{v: \mu_{\widetilde{C}}(v) \geq \lambda\right\}$ is the pseudo-inverse of $\mu_{\widetilde{C}}$. For $\widetilde{C}$ depicted in Fig. $1 \mathrm{~b} \mu_{\widetilde{C}}^{-1}(\lambda)=\widehat{C}+\rho+\xi(\lambda)=$ $\widehat{C}+\rho+\sigma \cdot\left(1-\lambda^{z_{c}}\right)$, where $\sigma$ is a given maximum allowed cost constraint violation (the slack), i.e. $\mu_{\widetilde{C}}^{-1}(0)=\widehat{C}+\rho+\xi(0)=$ $\widehat{C}+\rho+\sigma$. Replacing the left hand side of (46) by (11)-(21) 
and taking into account the forms of fuzzy intervals presented in Fig. 1 give the following mathematical programming model for the problem of computing a best necessarily soft feasible plan (44):

$$
\begin{aligned}
& (1-\lambda) \rightarrow \max \\
& \sum_{t \in[T]} \pi_{t}+c^{P} X_{T}+\Gamma \alpha+\sum_{t \in[T]} \gamma_{t} \leq \widehat{C}+\rho+\xi(\lambda),
\end{aligned}
$$

Constraints (12)-(15), (36)-(42).

Similarly as the model (33)-(42) for finding a best necessarily feasible plan the model (48)-(50) is nonlinear as well, now due to the terms $\alpha_{t}(\lambda), t \in[T]$, and $\xi(\lambda)$. In order to solve (48)-(50) we apply the binary search in $[0,1]$ previously used for (33)-(42). The only difference is that checking the feasibility of (34)-(41) is now replaced by checking the feasibility of (49)-(50). The number of such checkings is at most $\left\lceil\log \epsilon^{-1}\right\rceil$, where $\epsilon>0$ is a given accuracy. Furthermore, if $\alpha_{t}(\lambda), t \in[T]$, and $\xi(\lambda)$ are computed in polynomial time, then the running time of the binary search approach is polynomial.

The model (48)-(50) can be linearized by assuming that the uncertainty of the cumulative demands $\widetilde{D}_{t}, t \in[T]$, and the fuzzy goal $\widetilde{C}$ are modeled by the $L-R$ fuzzy intervals whose the left and right shape functions are such that $L_{t}=R_{t}=$ $L_{c}=R_{c}=L$, where $L_{t}, R_{t}$ and $L_{c}, R_{c}$ correspond to the cumulative demands $\widetilde{D}_{t}, t \in[T]$, and the goal $\widetilde{C}$, respectively. The above assumption leads to a compact linear programming model for the problem (44). A similar situation is for the forms of fuzzy intervals presented in Fig. 1. Indeed, $\alpha_{t}(\lambda)=\Delta_{t}$. $\left(1-\lambda^{z_{t}}\right), t \in[T]$, and $\xi(\lambda)=\sigma \cdot\left(1-\lambda^{z_{c}}\right)$ and assuming that $z_{c}=z_{t}=z$ for every $t \in[T]$, we have the same shape function $1-\lambda^{z}$ for the goal and the cumulative demands. Substituting $\Delta_{t} \cdot \Theta$ into $\alpha_{t}(\lambda)$ and $\sigma \cdot \Theta$ into $\xi(\lambda)$ in model (48)(50) we obtain its compact linearized counterpart:

$$
\begin{aligned}
& \Theta \rightarrow \max \\
& \sum_{t \in[T]} \pi_{t}+c^{P} X_{T} \\
& +\Gamma \alpha+\sum_{t \in[T]} \gamma_{t} \leq \widehat{C}+\rho+\sigma \Theta,
\end{aligned}
$$

Constraints (12)-(15),

$\alpha+\gamma_{t} \geq c^{I}\left(X_{t}-\left(\widehat{D}_{t}-\Delta_{t} \Theta\right)\right)-\pi_{t}, \quad t \in[T-1]$,

$\alpha+\gamma_{t} \geq c^{B}\left(\widehat{D}_{t}+\Delta_{t} \Theta-X_{t}\right)-\pi_{t}, \quad t \in[T-1]$,

$\alpha+\gamma_{T} \geq c^{I}\left(X_{T}-\left(\widehat{D}_{T}-\Delta_{T} \Theta\right)\right)$

$-b^{P}\left(\widehat{D}_{T}-\Delta_{T} \Theta\right)-\pi_{T}$,

$\alpha+\gamma_{T} \geq c^{B}\left(\widehat{D}_{T}+\Delta_{T} \Theta-X_{T}\right)$

$-b^{P} X_{T}-\pi_{T}$,

$\alpha, \gamma_{t} \geq 0, \pi_{t}$ unrestricted

$t \in[T]$

$x \in \mathbb{X}$,

$0 \leq \Theta \leq 1$,
Solving (51)-(60) yields a best necessarily soft feasible production plan $x^{*}$ and $\Theta_{\max }$. The degree of necessary $\Gamma$-soft feasibility of $\boldsymbol{x}^{*}$ can be computed as follows: $\mathrm{N}\left(\boldsymbol{x}^{*}\right.$ is $\Gamma$-FEAS $)=$ $1-\lambda_{\min }$, where $\lambda_{\min }=\left(1-\theta_{\max }\right)^{1 / z}$. Note also that solving (51)-(60) gives the same best necessarily soft feasible production plan $x^{*}$ for every $z>0$ with different degree of necessary $\Gamma$-soft feasibility.

\section{EXPERIMENTAL RESULTS}

In this section we present experiments illustrating the robust possibilistic approach (see Sec. IV) to the production planning problem (7) with cumulative demands under the possibilistic model of uncertainty (see Sec. IV-A) and compare this approach with the classical robust one that assumes the interval model of uncertainty (see Sec. III)

An instance $I$ of the problem (7) is built in the following way: the number of periods $T=25$; we are given one product and its the selling price $b^{P}$ is a random number uniformly distributed in $[100,150]$, the production, inventory and backordering costs are as follows: $c^{P}=0.8 b^{P}, c^{I}=0.05 c^{P}$ and $c^{B}=0.2 b^{P}$; the nominal value $\widehat{D}_{t}$ of $\widetilde{D}_{t}, t \in[T]$, is set to $\sum_{i \in[t]} \hat{d}_{i}$, where $\hat{d}_{i}$ is a nominal demand in period $i$ uniformly generated in $[700,1000]$. In order to ensure that the supports of $\widetilde{D}_{t}$ for every $t \in[T]$ are non-overlapping each maximum deviation $\Delta_{t}$ is a random number uniformly distributed in $\left[0.4 \min \left\{\hat{d}_{t}, \hat{d}_{t+1}\right\}, 0.5 \min \left\{\hat{d}_{t}, \hat{d}_{t+1}\right\}\right], t \in[T-1]$, for $t=T$, in $\left[0.4 \hat{d}_{T}, 0.5 \hat{d}_{T}\right]$. Each lower capacity limit $l_{t}$ is uniformly generated in $\left[0,1.1 \hat{d}_{t}\right]$ and the upper capacity limit $u_{t}$ is generated in $\left[l_{t}, 2 \hat{d}_{t}\right]$. There are no cumulative capacity limits $L_{t}$ and $U_{t}$. For both robust approaches we choose the robustness level $\Gamma=16$. In the robust possibilistic approach all the membership functions $\mu_{\widetilde{D}_{t}}, t \in[T]$, and $\mu_{\widetilde{C}}$ have the shape parameters such that $z_{c}=z_{t}=1$, i.e. $\widetilde{D}_{t}, t \in[T]$, are triangular fuzzy intervals and $\widetilde{C}$ is a trapezoidal fuzzy interval. The tolerance $\rho$ in the right hand side of the cost constraint, $\widehat{C}+\rho$, is set to $\rho=\hat{\rho} \cdot \widehat{C} / 100$ for $\hat{\rho} \in\{0 \%, 0.2 \%, 0.4 \%, \ldots, 10 \%\}$ and the slack $\sigma$ in the support of the fuzzy right hand side $\widetilde{C}, \widehat{C}+\rho+\sigma$, is set $\rho / 3$. We recall that $\widehat{C}$ denotes the cost of an optimal plan under the nominal cumulative demand scenario and $\mu_{\widetilde{C}}(\widehat{C}+\rho)=1$ and $\mu_{\widetilde{C}}(\widehat{C}+\rho+\sigma)=0$ (see Fig 1b).

Let $x_{I} \in \mathbb{X}$ be a solution (production plan) of instance $I$ of the problem (7) with respect to the aforementioned approaches, i.e. (10), (26) or (44). We use a Monte Carlo simulation to evaluate a posteriori the quality of $x_{I}$ in terms of its cost and the cost constraint violation under a randomly generated cumulative demand scenario $D$, according to the possibility distributions $\pi_{\widetilde{D}_{t}}, t \in[T]$. Namely, for each $\widetilde{D}_{t}, t \in[T]$ we generate its value as follows. We uniformly generate $\lambda$ in $[0,1]$ and then uniformly generate the value of $D_{t}$ in $\widetilde{D}^{\lambda}=\left[\widehat{D}-\Delta_{t}(1-\lambda), \widehat{D}+\Delta_{t}(1-\lambda)\right]$, which gives a scenario $\boldsymbol{D}=\left(D_{t}\right)_{t \in[T]}$ and in consequence a deterministic counterpart of (7), i.e. an instance of the problem (6). For this scenario $\boldsymbol{D}$ we compute the distance $\mathrm{d}\left(\boldsymbol{x}_{I}, \boldsymbol{D}\right)$ of the cost of $x_{I}$ from the instance optimal nominal cost $\widehat{C}_{I}, \mathrm{~d}\left(x_{I}, D\right)=$ 
$\left|\mathcal{C}\left(\boldsymbol{x}_{I}, \boldsymbol{D}\right)-\widehat{C}_{I}\right|$
$\left|\widehat{C}_{I}\right|$ tion $\mathrm{v}\left(\boldsymbol{x}_{I}, \boldsymbol{D}\right)$ of $\boldsymbol{x}_{I}, \mathrm{v}\left(\boldsymbol{x}_{I}, \boldsymbol{D}\right)=\max \left\{0, \mathcal{C}\left(\boldsymbol{x}_{I}, \boldsymbol{D}\right)-\left(\widehat{C}_{I}+\rho\right)\right\}$. We generate 1000 such random scenarios that form set $\mathbb{U}$. We determine the following quantities that express a posteriori evaluation of $\boldsymbol{x}_{I}$ computed, namely the average distance over $\mathbb{U}$, i.e. $\mathrm{d}_{\mathrm{avg}}\left(\boldsymbol{x}_{I}\right)=\underset{|\mathbb{U}|}{1} \sum_{\boldsymbol{D} \in \mathbb{U}} \mathrm{d}\left(\boldsymbol{x}_{I}, \boldsymbol{D}\right)$, the fraction of the scenarios in which $x_{I}$ violates the cost constraint $\#$ infeas $\left(\boldsymbol{x}_{I}\right)=\underset{|\mathbb{U}|}{1}\left|\left\{\boldsymbol{D} \in \mathbb{U}: \mathrm{v}\left(\boldsymbol{x}_{I}, \boldsymbol{D}\right)>0\right\}\right|$, and the average magnitude of the cost constraint violation $\mathrm{v}_{\text {avg }}(\boldsymbol{x})=$ ${ }_{|\mathbb{U}|} \sum_{\boldsymbol{D} \in \mathbb{U}} \mathrm{v}(\boldsymbol{x}, \boldsymbol{D})$.

Our computational experiments were made in the following way. For each $\hat{\rho} \in\{0 \%, 0.2 \%, 0.4 \%, \ldots, 10 \%\}$ we generated a set $\mathbb{I}$ of 100 random instances $\mathbb{I}=\left\{I_{1}, \ldots, I_{100}\right\}$ according to the scheme above described. For every instance $I \in \mathbb{I}$ we set $\rho=\hat{\rho} \cdot \widehat{C}_{I} / 100$ and $\sigma=\rho / 3$ and computed a best necessarily feasible production plan $x_{I}^{B N}$ by solving the linearized model (33)-(42), a best necessarily soft feasible production plan $x_{I}^{B N S}$ by solving (51)-(60), and a robust production plan $\boldsymbol{x}_{I}^{R}$ by solving (11)-(21). Note that $\boldsymbol{x}_{I}^{R}$ minimizes its maximum cost over scenario set $\mathcal{D} \cap \mathcal{U}$ and depends on neither $\rho$ nor $\sigma$ (see (11)-(21)). For solving the models we used IBM ILOG CPLEX 12.9 optimizer and the modeling package JuMP [23] embedded in the programming language Julia. For such plans computed we determined their average qualities over $\mathbb{I}$, i.e. for $x_{I}^{B N}$ :

$$
\begin{aligned}
\mathrm{d}^{\mathrm{BN}}(\hat{\rho}) & =\frac{1}{|\mathbb{I}|} \sum_{I \in[\mathbb{I}]} \mathrm{d}_{\mathrm{avg}}\left(\boldsymbol{x}_{I}^{\mathrm{BN}}\right), \\
\text { \#infeas }^{\mathrm{BN}}(\hat{\rho}) & =\begin{array}{c}
1 \\
|\mathbb{I}|
\end{array} \sum_{I \in[\mathbb{I} \mid]} \# \operatorname{infeas}\left(\boldsymbol{x}_{I}^{\mathrm{BN}}\right), \\
\mathrm{v}_{\mathrm{avg}}^{\mathrm{BN}}(\hat{\rho}) & =\frac{1}{|\mathbb{I}|} \sum_{I \in[\mathbb{I}]} \mathrm{v}_{\mathrm{avg}}\left(\boldsymbol{x}_{I}^{\mathrm{BN}}\right), \\
\mathrm{N}^{\mathrm{BN}}(\hat{\rho}) & =\frac{1}{|\mathbb{I}|} \sum_{I \in[\mathbb{I}]} \mathrm{N}\left(\boldsymbol{x}_{I}^{\mathrm{BN}} \text { is } \Gamma \text {-FEAS }\right) .
\end{aligned}
$$

For $x_{I}^{B N S}$ we computed (61)-(63) and $\mathrm{N}^{\mathrm{BNS}}(\hat{\rho})=$ ${ }_{|\mathbb{I}|}^{1} \sum_{I \in[\mathbb{I}]} \mathrm{N}\left(\boldsymbol{x}_{I}^{\mathrm{BNS}}\right.$ is $\Gamma$-FEAS $)$ instead of (64) and for $\boldsymbol{x}_{I}^{R}$ we determined only (61)-(63).

Fig. 2 presents average distances of the costs of the computed production plans from the instance optimal nominal costs for various $\hat{\rho}$. For robust plans $x_{I}^{R}$,s the distances are at the same level, approximately 0.078 , for every $\hat{\rho}$, because they do not depend on $\hat{\rho}$ and are only geared towards minimizing the worst case cost, whereas for best necessarily (soft) feasible production plans $\boldsymbol{x}_{I}^{B N}$ 's ( $\boldsymbol{x}_{I}^{B N S}$ 's) the (flexible) cost constraint and information about plausibility of cumulative demands scenarios are additionally taken into account while they are computed. Thus evident differences in the distances between $\boldsymbol{x}_{I}^{R}$ 's and $\boldsymbol{x}_{I}^{B N}$ 's $\left(\boldsymbol{x}_{I}^{B N S}\right.$ 's) have been revealed. Note also that the (flexible) cost constraint is tight at some $\lambda_{\min }$ 's for $\lambda_{\min } \in[0,1), \lambda_{\min }=1-\Theta_{\max }$, (see Fig. 5 for average $\Theta_{\max }$ 's).

Fig. 3 and 4 show fractions of scenarios in which production plans computed violate the cost constraint and average magni-

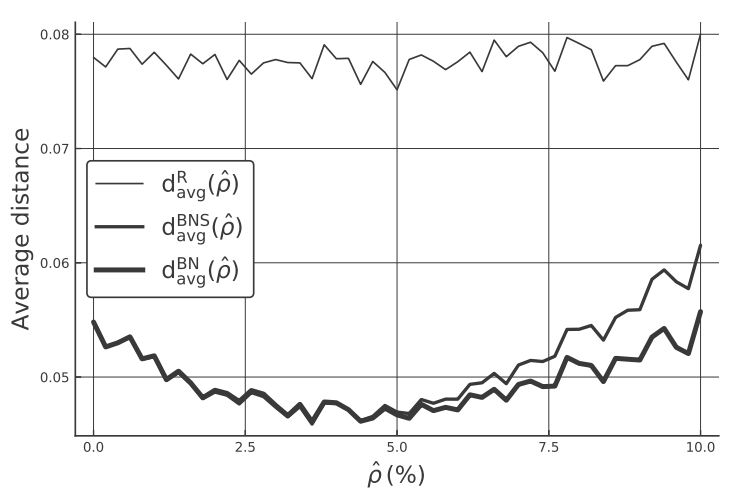

Fig. 2. Average distances of costs of production plans from the optimal instance nominal costs for various $\hat{\rho}$.

tudes of the cost constraint violation. In both figures the graphs for best necessarily feasible plans and best necessarily soft feasible ones overlap, however the numerical results that are behind slightly differ. Obviously, for $\hat{\rho}=0 x_{I}^{B N}$, s and $x_{I}^{B N S}$, s violate the cost constraint for all the generated scenarios, since it has the form of $\widehat{C}_{I}(\rho=0)$. In this case their robustness are very weak. It is the first extreme case, where the attention is paid on the costs of plans computed. For $\hat{\rho}>10 \% \boldsymbol{x}_{I}^{B N}$ 's and $x_{I}^{B N S}$ 's are feasible under almost all scenarios and it is the second extreme case, where there has been a shift of emphasis from the costs to the robustness of the plans. A compromise situation can be observed around $\hat{\rho} \approx 5 \%$ in Fig. 3 and 4 and also in Fig. 2. Thus for $\hat{\rho} \approx 5 \% \boldsymbol{x}_{I}^{B N}$, s and $\boldsymbol{x}_{I}^{B N S}$, s can be seen as best choices, since they are compromise ones. Furthermore their performance is better than the performance of robust plans with respect to all the criteria (61)-(63) examined, since information about a possibility distribution of cumulative demand scenarios, induced by fuzzy cumulative demands, is exploited while they are build. Accordingly, the main conclusion can be drawn from the experimental results that taking the possibilistic information into account can improve the quality of the obtained production plans.

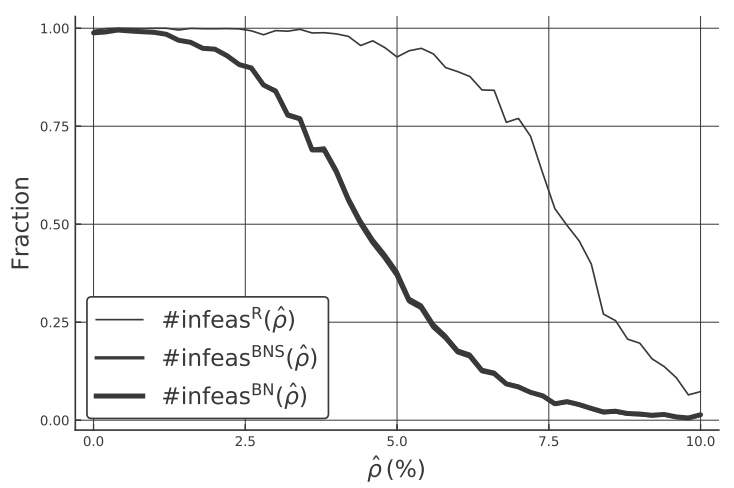

Fig. 3. Fractions of scenarios in which production plans violate the cost constraint for various $\hat{\rho}$. 


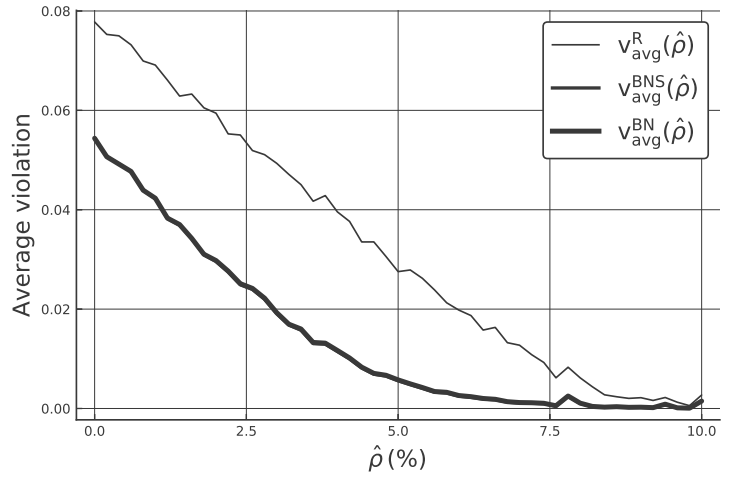

Fig. 4. Average magnitudes of the cost constraint violation for various $\hat{\rho}$.

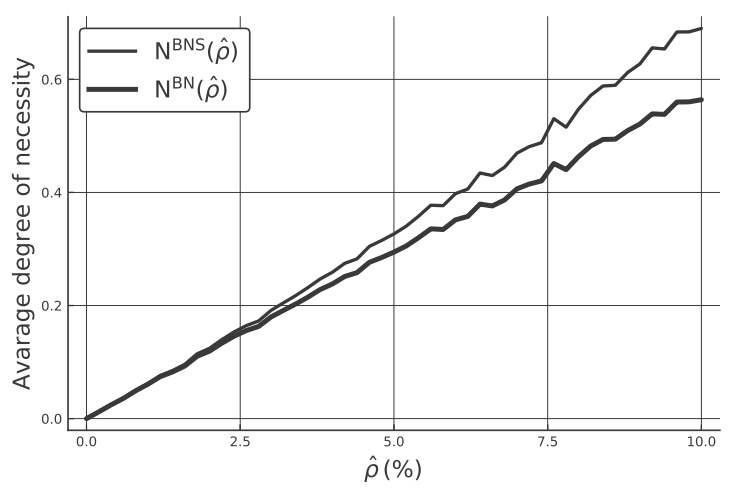

Fig. 5. Average degrees of necessity that plans are $\Gamma$-(soft) feasible for various $\hat{\rho}$.

\section{CONCLUSIONS}

In this paper we dealt with a version of the capacitated single-item lot sizing problem with backordering under uncertain cumulative demands modeled by fuzzy intervals prescribed whose membership functions are regarded as possibility distributions for their values. The distributions induce a possibility distribution in scenario set $\mathcal{U}$ that describes plaussible cumulative demand scenarios and is an upper bound on the unknown probability one. We applied the possibilistic approach [17], to the problem under consideration, which exploits information about a possibility distribution in $\mathcal{U}$ while a robust production plan is constructed and allows decision makers to control a price of robustness by specifying the (flexible) cost constraint, unlike the traditional robust approach [13], [14] that only assumes that scenarios belong to $\mathcal{U}$. Therefore, the possibilistic approach provides a robust plan that is a compromise one, because it optimizes against plausible scenarios and its costs in plausible scenarios are not too far from the cost an optimal plan in the nominal scenario. Indeed, we confirmed the above fact experimentally, namely the experiments performed illustrate, among others, that applying the possibilistic approach leads to production plans with lower costs over a set of scenario randomly generated according to a possibilistic distribution in $\mathcal{U}$. Furthermore, we proposed polynomial linear programming based methods for finding such robust production plans, showing in this way that introducing uncertainty in the cumulative demands in the possibilistic setting does not make our problem much computationally harder than its deterministic counterpart.

\section{REFERENCES}

[1] J. R. T. Arnold, S. N. Chapman, and L. M. Clive, Introduction to Materials Management, 7th ed. Prentice Hall, 2011.

[2] H. Fargier and C. Thierry, "The Use of Possibilistic Decision Theory in Manufacturing Planning and Control: Recent Results in Fuzzy Master Production Scheduling," in Advances in Scheduling and Sequencing under Fuzziness, R. Slowiński and M. Hapke, Eds. Springer-Verlag, 2000, pp. 45-59.

[3] J. Mula, R. Poler, and J. P. Garcia-Sabater, "Material Requirement Planning with fuzzy constraints and fuzzy coefficients," Fuzzy Sets and Systems, vol. 159, pp. 783-793, 2007.

[4] R. Tavakkoli-Moghaddam, M. Rabbani, A. H. Gharehgozli, and N. Zaerpour, "A Fuzzy Aggregate Production Planning Model for Make-toStock Environments," in Proceedings of the International Conference on Industrial Engineering and Engineering Management, 2007, pp. 16091613.

[5] B. Grabot, L. Geneste, G. Reynoso-Castillo, and S. Vérot, "Integration of uncertain and imprecise orders in the MRP method," Journal of Intelligent Manufacturing, vol. 16, pp. 215-234, 2005.

[6] R. Guillaume, C. Thierry, and B. Grabot, "Modelling of ill-known requirements and integration in production planning," Production Planning and Control, vol. 22, pp. 336-352, 2011.

[7] D. Peidro, J. Mula, R. Poler, and F. C. Lario, "Quantitative models for supply chain planning under uncertainty: a review," The International Journal of Advanced Manufacturing Technology, vol. 43, pp. 400-420, 2009.

[8] Y. Pochet and L. A. Wolsey, Production Planning by Mixed Integer Programming. Springer-Verlag, 2006.

[9] P. Kouvelis and G. Yu, Robust Discrete Optimization and its Applications. Kluwer Academic Publishers, 1997.

[10] D. Bertsimas and A. Thiele, "A robust optimization approach to inventory theory," Operations Research, vol. 54, pp. 150-168, 2006.

[11] M. A. Abdel-Aal, "A robust capacitated lot sizing problem with setup times and overtime decisions with backordering allowed under demand uncertainty," IFAC-PapersOnLine, vol. 52, pp. 589 - 594, 2019.

[12] D. Dubois and H. Prade, Possibility theory: an approach to computerized processing of uncertainty. New York: Plenum Press, 1988.

[13] D. Bertsimas and M. Sim, "Robust discrete optimization and network flows," Mathematical Programming, vol. 98, pp. 49-71, 2003.

[14] — , "The price of robustness," Operations research, vol. 52, pp. 35-53, 2004.

[15] M. Fischetti and M. Monaci, "Light Robustness," in Robust and Online Large-Scale Optimization: Models and Techniques for Transportation Systems. Springer-Verlag, 2009, pp. 61-84.

[16] A. Schöbel, "Generalized light robustness and the trade-off between robustness and nominal quality," Mathematical Methods of Operations Research, vol. 80, pp. 161-191, 2014.

[17] A. Kasperski and P. Zieliński, "A (Soft) Robustness for Possibilistic Optimization Problems,", in 2019 IEEE International Conference on Fuzzy Systems, FUZZ-IEEE 2019, 2019, pp. 1-6.

[18] W. A. Lodwick and J. Kacprzyk, Eds., Fuzzy Optimization - Recent Advances and Applications, ser. Studies in Fuzziness and Soft Computing. Springer-Verlag, 2010, vol. 254.

[19] R. Guillaume, A. Kasperski, and P. Zieliński, "Production planning under demand uncertainty: a budgeted uncertainty approach," in Operations Research Proceedings 2019, to appear.

[20] D. Dubois, "Possibility theory and statistical reasoning," Computational Statistics and Data Analysis, vol. 51, pp. 47-69, 2006.

[21] D. Dubois, H. Fargier, and P. Fortemps, "Fuzzy scheduling: Modelling flexible constraints vs. coping with incomplete knowledge," European Journal of Operational Research, vol. 147, pp. 231-252, 2003.

[22] D. Dubois, L. Foulloy, G. Mauris, and H. Prade, "Probability-possibility transformations, triangular fuzzy sets and probabilistic inequalities,' Reliable Computing, vol. 10, pp. 273-297, 2004.

[23] M. Lubin and I. Dunning, "Computing in operations research using julia," INFORMS Journal on Computing, vol. 27, pp. 238-248, 2015. 